\title{
The Acute Effect of Meal Timing on the Gut Microbiome and the Cardiometabolic Health of the Host: A Crossover Randomized Control Trial
}

\author{
Rodanthi Thalia Papadopoulou Maria Rafailia Theodorou Chi San leong \\ Katrina Ballantyne Danae Marshall Anais Verney Marine Roig Ben Nichols \\ Konstantinos Gerasimidis
}

Human Nutrition, School of Medicine, Dentistry and Nursing, University of Glasgow, New Lister Building, Glasgow, UK

\section{Keywords}

Meal timing - Gut microbiome - Short-chain fatty acids .

Cardiovascular diseases - Western diet

\begin{abstract}
Purpose: The interaction of diet with gut microbiome has been implicated in the onset of cardiovascular disease. The gut microbiome displays diurnal rhythms, which may be influenced by meal timing. Objective: This study aimed to investigate the effect of the timing of main meal consumption on the microbiome and cardiometabolic biomarkers of the host. Methods: Seventeen healthy adults randomly consumed an isocaloric diet for 7 days, twice, by alternating lunch with dinner meals, and with a 2-week washout in-between. Sixty percent of the participants' daily energy requirements was consumed either as lunch or dinner, respectively. Meals were provided free to the participants. All fecal samples produced the last 3 days of each intervention were collected and analyzed for microbial profiling (16S rRNA gene amplicon sequencing), quantitative estimation of representative bacterial groups (qPCR) of the gut microbiome, and the output of short-chain fatty acids (SCFA) in feces. Fasted blood samples were analyzed for low-grade inflammatory biomarkers, blood lipids, insulin, and glucose levels.
\end{abstract}

Cumulative energy loss in feces was measured over the collection period using bomb calorimetry. Results: Meal timing had no significant effects on fecal SCFA output, energy loss in feces, microbial community profiling, and bacterial species relative abundance. The absolute concentration of Escherichia coli was significantly higher after the large lunch intervention ( $p=0.02$ ). No effects on blood biomarkers of cardiometabolic health were observed. Conclusions: In a well-controlled study, main meal timing displayed minimal acute effects on the gut microbiome composition, its dietrelated function, and blood biomarkers of cardiometabolic health.

(C) 2020 The Author(s)

Published by S. Karger AG, Basel

\section{Introduction}

The gut microbiome and the host are intrinsically linked to each other. There is a growing body of evidence drawing associations between disruptions to the microbiome (dysbiosis) and noncommunicable diseases such as inflammatory bowel disease [1] and, beyond the gastro-

R.T. Papadopoulou and M.R. Theodorou are co-first authors. karger@karger.com www.karger.com/anm

Karger $\stackrel{\text { ' }}{5}$

GOPEN ACCESS
(C) 2020 The Author(s)

Published by S. Karger AG, Basel

This article is licensed under the Creative Commons Attribution 4.0 International License (CC BY) (http://www.karger.com/Services/ OpenAccessLicense). Usage, derivative works and distribution are permitted provided that proper credit is given to the author and the original publisher.
Konstantinos Gerasimidis

Human Nutrition, School of Medicine, College of Medical, Veterinary and Life Sciences University of Glasgow

Glasgow G31 2ER (UK)

konstantinos.gerasimidis@glasgow.ac.uk 
intestinal tract, cardiovascular disease (CVD) [2]. The exact mechanisms by which diet influences the onset of CVD or determines cardiometabolic health are incompletely known, but emerging research hints at a complex diet-microbiome interaction [3]. For example, a high-fat diet can reduce the abundance of bacterial species associated with good cardiometabolic health, and certain Gramnegative bacteria may promote type 2 diabetes through endotoxin-induced inflammation, whereas fermentation of dietary fiber produces short-chain fatty acids (SCFA) which have beneficial effects on appetite regulation, de novo lipogenesis, and energy substrate metabolism [2, 4].

Interestingly though it may not only be the macronutrient composition of the diet that influences the gut microbiome and by extension the CVD risk. Recent research has begun to unveil the complex relationship between meal timing and frequency, diurnal rhythm, and the microbiome. In a cross-sectional study, the human fecal microbiome and diet-produced bacterial metabolites were associated with time of day, eating frequency, percentage of energy consumed early in the day, and overnight-fast duration [5]. The factors which influence these diurnal fluctuations in the gut microbiome are still unknown and understudied. Previous research suggests that gastrointestinal bacteria have their own intrinsic biological clocks, as well as responding to the host's circadian rhythm [6, 7]. In a small study, Thaiss et al. [8] demonstrated, in mice and in humans, that jet-lag-induced microbiome dysbiosis causes weight gain, glucose intolerance, and an increase in body adiposity, symptoms which were transferrable to non-jet-lagged mice with fecal transplantation.

The human diurnal or circadian rhythm is entrained by light and the timing of food consumption. If the bacterial clock is also entrained by the same stimulants as humans, it could be hypothesized that the time of food consumption would be a modifier for the gut microbiome and by extension of risk of noncommunicable diseases associated with the former. This is interesting to study as previous research has shown that consuming a higher proportion of energy intake at midday may have beneficial health effects, such as greater weight loss and better insulin sensitivity [9], and energy intake in the evening might be a risk factor for obesity [10]. Whether the microbiome is implicated in this process is unknown, and it is difficult to establish causation via early data from association studies [5]. A recent intervention study in 10 healthy volunteers explored the effect delaying lunch by 3:30 $\mathrm{h}$ might have on the fecal and salivary microbiome. The authors observed significant effects on the diversity and bacterial relative abundance of the salivary microbi- ome but no influence effects on the microenvironment of a spot fecal sample [11]. To the best of our knowledge, there are no well-designed intervention studies which have investigated the effect swapping lunch with dinner would have on the gastrointestinal microbiome, diet-related metabolism, gut physiology, and host cardiometabolic health.

Considering the recent hypotheses generated from association studies and animal experiments, the current study aimed to investigate the acute effects of the time of main meal consumption on the gut microbiome and host cardiometabolic health.

\section{Materials and Methods}

This study is a crossover randomized control trial in healthy adults ( $>18$ years) recruited by means of advertisement. Eligible participants were screened using a health check questionnaire which included questions about current and past medical history, use of medication, and previous major gastrointestinal operations. Those with a history of major gut surgery, antibiotic use during the last 3 months, weight change during the past month $( \pm 2 \mathrm{~kg})$, and presence of chronic illness (defined as illness requiring regular visits to a doctor or regular use of medication) and pregnant women were excluded.

\section{Dietary Interventions}

Participants consumed the same diet twice, but each time under different meal timing condition. Each intervention lasted for a week. All participants undertook both dietary interventions with a 2-week "washout" period in-between, where participants maintained their habitual diet (Fig. 1). Participants were allocated to their first intervention using simple randomization (i.e., participants flipped a coin). The duration of the intervention and washout was based on evidence that the human gut microbiome is rapidly altered within just 3 days of diet change $[12,13]$. The dietary interventions were based on a typical Western diet consisting of $50 \%$ carbohydrate, $35 \%$ fat, and $15 \%$ protein (see online suppl. material 1; see www.karger.com/doi/10.1159/000510646 for all online suppl. material). The 2 dietary interventions were identical, in terms of meal types and the day during the week these were consumed, with the only difference being the time of the main meal consumption. Participants were asked to consume $60 \%$ of their daily energy requirements either as lunch, between 12:30 and 13:30, or as dinner, between 19:30 and 20:30, each day during their 2 dietary interventions. Breakfast accounted for $15 \%$ of their total daily energy requirements, and a morning and evening snack each accounted for $5 \%$ energy requirements. Fifteen percent of their energy requirements were allocated as a light meal, either as dinner or lunch, depending on the timing of the main meal. The participants were provided with food and meal lists from which they chose their preferred ones to consume during the interventions. Each participant consumed exactly the same food for both interventions and on the same days. All meals were purchased and provided to the participants free of charge to encourage compliance and control dietary intake. Participants were asked to complete their meals within a maximum of $30 \mathrm{~min}$ of serving. During the 


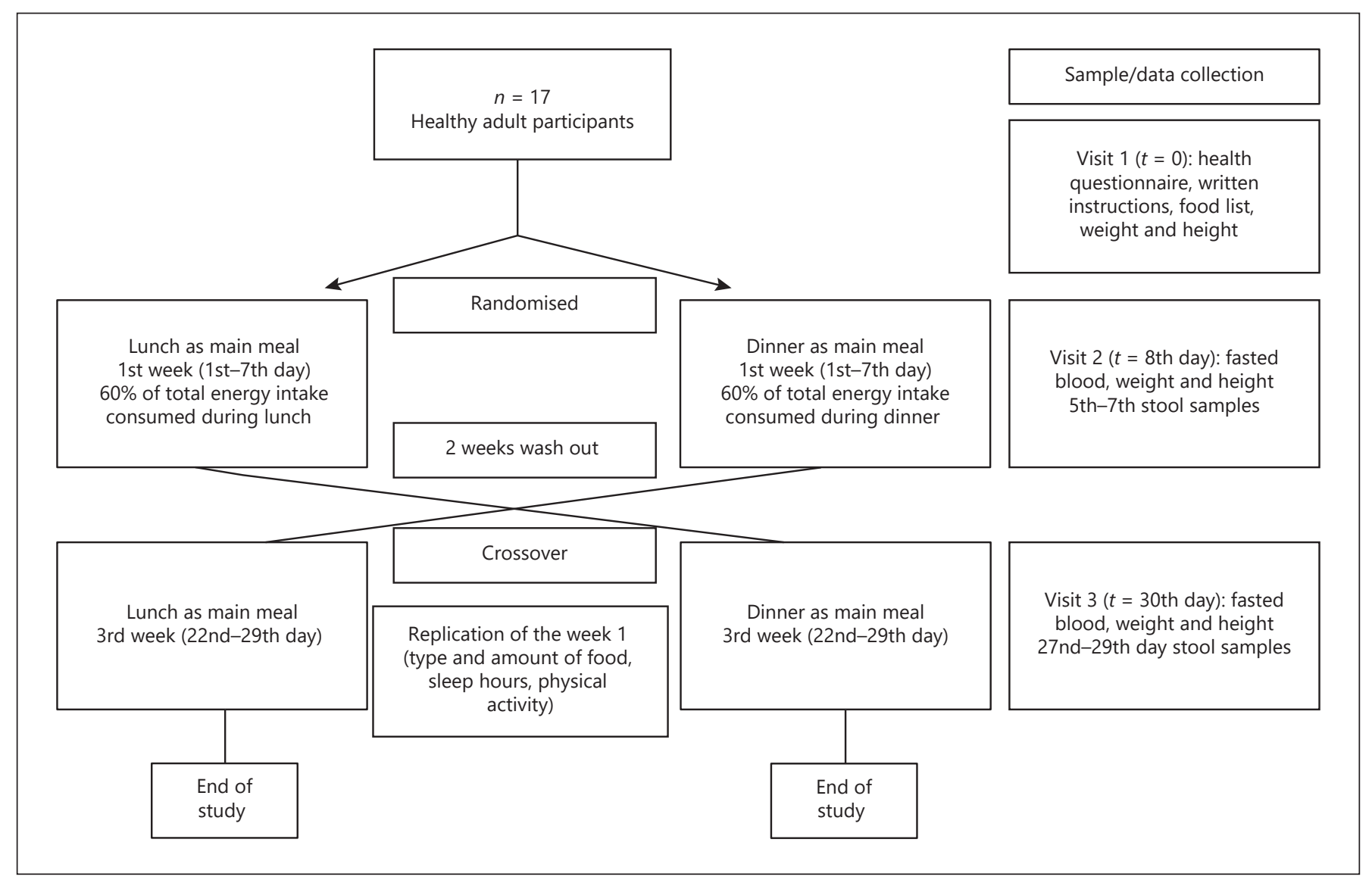

Fig. 1. Design of the crossover randomized controlled trial. $t$, timepoint.

study course, compliance to the dietary intervention was monitored against a check list of the meals the participants were provided. Individual dietary plans were created for each participant separately, based on their daily energy requirements. The energy requirements of each participant were calculated using the Schofield equation accounting for individual physical activity levels. Measurements of weight and height were obtained before and after each intervention at the research metabolic unit in Human Nutrition, University of Glasgow. The study was approved by the MLVS Research Ethics Committee at the University of Glasgow (reference No. 200170046). All participants provided written informed consent and were reimbursed with GBP 100 shopping vouchers or a donation to a charity of their choice.

The study was conducted according to the criteria set by the World Medical Association Declaration of Helsinki. Additionally, the study was registered with clinicaltrials.gov (NCT03949543).

\section{Fecal Sample Collection}

All stools produced from 12:00 noon on the fifth day of the intervention until 12:00 noon on the eighth day of the intervention (cumulative 3-day stool collection) were collected during both interventions. The entire bowel movement was collected using provided stool collection kits. Immediately after passage, each sample was transferred to the laboratory under anaerobic conditions (Oxoid $^{\mathrm{TM}}$ AnaeroGen ${ }^{\mathrm{TM}}$ ) using a cool bag and ice packs and within
$2 \mathrm{~h}$ of defecation. All samples were weighted, homogenized, and stored for downstream analysis. The shape and texture of the samples were rated using the Bristol Stool Scale. Fecal pH was measured in 1:3 w/v slurries and fecal ammonia with an ammonia analyzer (HI 93715; Hanna, Bedfordshire, UK). Fecal water content was calculated from the dry matter after freeze-drying.

\section{Fecal Microbiome}

Extraction of genomic DNA was performed using the PowerSoil ${ }^{\circledR}$ DNA isolation kit which includes an initial bead-beating step (FastPrep-24 ${ }^{\mathrm{TM}}$ ), according to the manufacturer's instructions (Qiagen, Manchester, UK). Purity was checked on 1\% agarose gel electrophoresis, and the concentration of nucleic acids was measured with Nanodrop ${ }^{\mathrm{TM}}$ and Qubit. Quantification (16S rRNA gene copy number/g stool) of total and representative dominant and subdominant bacterial groups of the human gut microbiome (Bacteroides/Prevotella, Clostridium leptum cluster, Clostridium coccoides cluster, Bifidobacterium, Lactobacillus, and Escherichia coli) was carried out with quantitative PCR using TaqMan ${ }^{\mathrm{TM}}$ chemistry [14] (online suppl. material 2). Absolute quantification was performed against serial dilutions of bacterial DNA standards obtained from the pure strain. The V4 region of the $16 \mathrm{~S}$ rRNA gene of the last sample provided by each participant and for each intervention was amplified, and sequencing was performed (MiSeq, Illumina, Essex, UK) using $2 \times 250$-bp paired-end reads [1]. 
Table 1. Difference in the characteristics and energy content in feces between the 2 interventions

\begin{tabular}{lccc}
\hline & Large dinner & Large lunch & $p$ value \\
\hline Characteristics of feces & & & \\
$\quad$ Fecal pH & $6.7(0.5)$ & $6.6(0.5)$ & 0.69 \\
$\quad$ Bristol stool chart & $3.1(1.4)$ & $4.2(1.4)$ & 0.24 \\
$\quad$ Total stool output over 3 days, g & $275(147)$ & $311(249)$ & 0.46 \\
$\quad$ Fecal water content, \% & $70.6(8.6)$ & $69.8(6.1)$ & 0.63 \\
Energy content in feces & & & \\
$\quad$ Average of all samples per participant, kcal/g & $1.3(0.3)$ & $1.3(0.4)$ & 0.83 \\
$\quad$ Output over 3-day collection, kcals & $333(174)$ & $335(165)$ & 0.97 \\
$\quad$ Concentration of output over 3-day collection, kcal/g & $1.3(0.3)$ & $1.3(0.4)$ & 0.64 \\
\hline
\end{tabular}

Data are presented with means (SD); $p$ value for paired $t$ test comparison between the 2 interventions.

\section{Fecal SCFA}

Fecal SCFA and branched-chain fatty acids were quantified by gas chromatography in diethyl ether extracts [14]. Quantification was performed against authentic standards, and 2-ethylbutyric acid was used as the internal standard.

\section{Energy Loss in Feces}

Energy excreted in feces collected over the last 3 days of the intervention was measured using the bomb calorimeter (Parr 6100) in dry feces $(500 \mathrm{mg})$. The bomb calorimeter was calibrated daily with benzoic acid tablets. Analysis was performed in duplicate or until the coefficient of variation $(\% \mathrm{CV})$ between replicates was $<5 \%$. The difference between dietary energy intake and energy loss in feces was calculated.

\section{Blood Lipid Analysis}

Fasted venous blood samples were collected at 8 a.m. the day after the intervention, in ethylenediaminetetraacetic acid tubes. Tubes containing blood samples were immediately placed on ice and then centrifuged at $4^{\circ} \mathrm{C}$, at 3,000 rpm for $15 \mathrm{~min}$. Plasma was dispensed in $0.5-\mathrm{mL}$ tubes and kept at $-80^{\circ} \mathrm{C}$ until analysis. High sensitivity interleukin-6 (IL-6) (Invitrogen, Cat\# BMS213HS) and high sensitivity tumor necrosis factor- $\alpha$ (TNF- $\alpha$ ) (Invitrogen, Cat\# BMS223HS) and insulin (Mercodia, Cat\# 10-1113-0) were measured using ELISA kits. Plasma glucose, total cholesterol, HDL cholesterol, LDL cholesterol, triglyceride, and high-sensitivity C-reactive protein (CRP) were measured at the Queen Elizabeth University Hospital accredited Clinical Biochemistry laboratories.

\section{Bioinformatics}

Operational taxonomic units (OTUs) were used to analyze the gut microbial (i.e., bacterial) composition. OTUs were generated from $16 \mathrm{~S}$ rRNA amplicon sequencing data using a modified version of the VSEARCH pipeline (https://github.com/torognes/ vsearch/wiki/VSEARCH-pipeline). The paired fastq files were combined, and a maximum error rate of $0.5 \mathrm{bp}$ per read was used for the quality filtering step. Sequences longer than $275 \mathrm{bp}$ and shorter than 225 bp were filtered out. The fastq files were pooled together, dereplicated, all singleton sequences were removed, and sequences were then preclustered at $98 \%$. Chimera identification
Table 2. Difference in the concentration and output of fecal SCFA between the 2 interventions

\begin{tabular}{|c|c|c|c|}
\hline & Large dinner & Large lunch & $p$ value \\
\hline \multicolumn{4}{|c|}{ Average of all samples per participant, $\mu \mathrm{mol} / \mathrm{g}$} \\
\hline Acetate & $14,453(10,541)$ & $13,348(10,197)$ & 0.62 \\
\hline Propionate & $3,570(2,172)$ & $3,615(1,797)$ & 0.95 \\
\hline Iso-butyrate & $298(167)$ & $290(204)$ & 0.86 \\
\hline Butyrate & $3,308(3,107)$ & $2,787(2,222)$ & 0.33 \\
\hline Iso-valeric & $374(222)$ & $358(257)$ & 0.80 \\
\hline Valeric & $377(271)$ & $369(302)$ & 0.87 \\
\hline Iso-hexanoic & $18.2(15)$ & $14.7(11.8)$ & 0.31 \\
\hline Hexanoic & $113(130)$ & $116(98)$ & 0.68 \\
\hline Heptanoic & $17.3(18.3)$ & $15.3(11.7)$ & 0.49 \\
\hline Octanoic & $6.9(3)$ & $14.2(21.4)$ & 0.20 \\
\hline \multicolumn{4}{|c|}{ Output over 3-day collection, $\mu \mathrm{mol}$} \\
\hline Acetate & $32,378(2,700)$ & $34,353(30,175)$ & 0.76 \\
\hline Propionate & $8,133(5,792)$ & $9,479(11,406)$ & 0.52 \\
\hline Iso-butyrate & $626(282)$ & $696(429)$ & 0.80 \\
\hline Butyrate & $7,496(8,415)$ & $7,188(6,602)$ & 0.84 \\
\hline Iso-valeric & $775(393)$ & 849 (557) & 0.76 \\
\hline Valeric & $817(541)$ & $918(730)$ & 0.34 \\
\hline Iso-hexanoic & $41.9(44.2)$ & $36.3(30.4)$ & 0.09 \\
\hline Hexanoic & $224(209)$ & $279(230)$ & 0.31 \\
\hline Heptanoic & $33.8(36.3)$ & $35.2(29.2)$ & 0.31 \\
\hline Octanoic & $15.0(8.6)$ & $35.9(60.7)$ & 0.65 \\
\hline \multicolumn{4}{|c|}{ Concentration of output over 3 -day collection, $\mu \mathrm{mol} / \mathrm{g}$} \\
\hline Acetate & $111(32)$ & $112(40)$ & 0.90 \\
\hline Propionate & $29.1(11.9)$ & $30(17)$ & 0.79 \\
\hline Iso-butyrate & $2.6(0.9)$ & $2.8(1.9)$ & 0.55 \\
\hline Butyrate & $24.5(12.2)$ & $23.5(9.3)$ & 0.70 \\
\hline Iso-valeric & $3.2(1.7)$ & $3.2(1.8)$ & 0.54 \\
\hline Valeric & $1.9(1.0)$ & $1.9(1.0)$ & 0.91 \\
\hline Iso-hexanoic & $0.2(0.1)$ & $0.1(0.1)$ & 0.42 \\
\hline Hexanoic & $1.0(1.0)$ & $1.1(0.9)$ & 0.53 \\
\hline Heptanoic & $0.2(0.2)$ & $0.2(0.1)$ & 0.78 \\
\hline Octanoic & $0.1(0.1)$ & $0.1(0.2)$ & 0.28 \\
\hline
\end{tabular}

Data are presented as means (SD); $p$ value for paired $t$ test comparison between the 2 interventions. SCFA, short-chain fatty acids. 


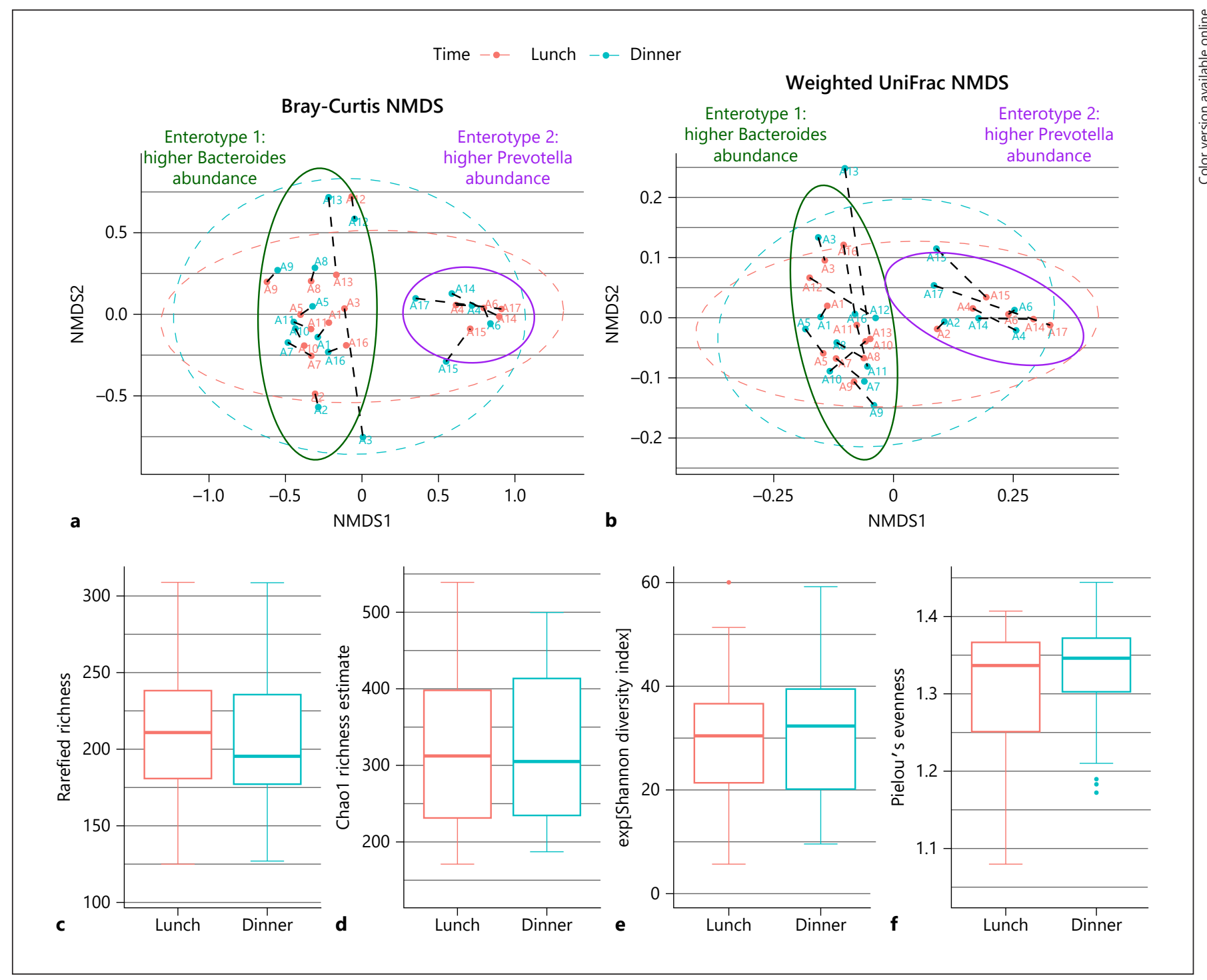

Fig. 2. Effect of timing of main meal consumption on the gut microbiome of healthy volunteers. a NMDS using Bray-Curtis distances of OTU community structure. b NMDS using weighted Unifrac distance analysis. c Rarefied richness. d Chaol index. e Exponential of Shannon index $(\mathrm{eH})$. f Pielou's evenness. NMDS, nonmetric multidimensional scaling; OTU, operational taxonomic unit.

and removal was carried out using the VSEARCH implementation of the UCHIME de novo algorithm. Following this, a secondary chimera detection and removal step was applied, this time using the UCHIME reference-based method and the "Gold" ChimeraSlayer reference dataset. OTUs were then generated by clustering the remaining sequences at $97 \%$. OTUs were taxonomically classified to genus level using the RDP naive Bayesian classifier method implemented in the dada2 $\mathrm{R}$ package.

Statistical Analysis

Statistical analysis was performed using Minitab 17 and R. Analysis of the microbiome data was performed using the phylo- seq (NMDS plots) and vegan (permutation ANOVA, richness, diversity, and evenness measurements) packages in R. Significantly different OTUs and genera were identified using paired $t$ tests on the log proportional abundances of each OTU or genus with Benjamini-Hochberg corrections for multiple testing applied to the resultant $p$ values. As baseline samples were not collected, we performed differential analysis at the end of the 2 interventions. Significant thresholds were applied at $p<0.05$ for adjusted $p$ values. In the absence of pilot data, no formal power calculation was carried out prior to study initiation. However, decision to extend or discontinue the study was made following interim analysis of the data of the first participants. 


\section{Results}

\section{Participants}

Seventeen participants took part in this study ( $8 \mathrm{fe}-$ males and 9 males; mean [SD] age of 26.2 [3.8], min.-max. 23-39 years; BMI of 24.3 [5.3] kg/m²). By these 17 participants, a total of 81 stools were produced and collected: 38 (47\%) over the large lunch intervention and 43 (53\%) over the large dinner intervention. Daily energy intake varied among participants, ranging from 1,801 to 3,478 kcal with an average (SD) of 2,606 (513) kcal. There was no significant mean weight change (mean [SD]: 0.29 [0.6] $\mathrm{kg} ; p=0.053$ ) during the large lunch intervention, but following the large dinner intervention, a significant increase in average (SD) body weight by $0.47(0.72) \mathrm{kg}(p=$ $0.016)$ was observed.

\section{Stool Characteristics}

A high variation in the total amount of feces produced per day was observed among individuals (\%CV:64\%), but the corresponding intraindividual variation was much lower (average $\% \mathrm{CV}$ of participants: $42 \%$ ). Total stool output (i.e., total weight of feces) produced over the 3 days of collection did not differ between the 2 interventions and neither did the Bristol Stool Scale rating, fecal $\mathrm{pH}$, and fecal water content (\%) (Table 1).

\section{Energy Output in Feces}

Participants lost on average 5.5\% of their daily energy intake in feces with no differences between the 2 interventions (lunch $5.2 \%$ vs. dinner $5.8 \%, p=0.83$ ). Similarly, the time of main meal consumption did not significantly associate with fecal energy output or content (Table 1).

\section{Fecal SCFA}

Differences in the production of fecal SCFA between interventions were expressed using 4 different approaches: (a) total 3-day fecal output, (b) average of all samples per participant, (c) concentration of 3-day output, and (d) as average relative (\%) abundance. Regardless of the way data were expressed, fecal SCFA were not significantly different between the 2 interventions (Table 2).

\section{Fecal Microbiome}

The last sample provided by each participant and for each intervention was sequenced using the $\mathrm{V} 4$ region of the 16S rRNA gene. Thirty-four samples with $>10,000$ reads were brought forward in the final analysis. The total number of OTUs identified across all samples was $n=$ 922. There were no significant differences between the 2
Table 3. Difference in the concentration and output of major bacterial groups between the 2 interventions

\begin{tabular}{|c|c|c|c|}
\hline & $\begin{array}{l}\text { Large } \\
\text { dinner }\end{array}$ & $\begin{array}{l}\text { Large } \\
\text { lunch }\end{array}$ & $\begin{array}{l}p \\
\text { value }\end{array}$ \\
\hline \multicolumn{4}{|c|}{ Average concentration ${ }^{\mathrm{a}}$ of all samples per participant } \\
\hline Total bacteria & $10.5(0.3)$ & $10.5(0.4)$ & 0.67 \\
\hline Bacteroides/Prevotella & $9.4(0.4)$ & $9.5(0.3)$ & 0.20 \\
\hline Bifidobacterium spp. & $8.3(0.4)$ & $8.3(0.4)$ & 0.93 \\
\hline C. leptum group & $9.6(0.8)$ & $9.5(0.3)$ & 0.65 \\
\hline C. coccoides group & $8.7(0.4)$ & $8.7(0.3)$ & 0.93 \\
\hline E. coli & $7.0(0.7)$ & $7.5(0.7)$ & 0.02 \\
\hline \multicolumn{4}{|l|}{ Output over 3-day collection } \\
\hline Total bacteria & $13.0(0.5)$ & $12.8(0.5)$ & 0.65 \\
\hline Bacteroides/Prevotella & $11.8(0.5)$ & $11.9(0.4)$ & 0.22 \\
\hline Bifidobacterium spp. & $10.7(0.5)$ & $10.7(0.4)$ & 0.99 \\
\hline C. leptum group & $11.9(0.6)$ & $11.8(0.4)$ & 0.62 \\
\hline C. coccoides group & $11.1(0.5)$ & $11.1(0.4)$ & 0.86 \\
\hline E. coli & $9.5(0.8)$ & $9.9(0.7)$ & 0.05 \\
\hline \multicolumn{4}{|c|}{ Concentration $^{\mathrm{a}}$ of output over 3-day collection } \\
\hline Total bacteria & $12.5(0.6)$ & $12.5(0.7)$ & 0.18 \\
\hline Bacteroides/Prevotella & $11.5(0.5)$ & $11.5(0.4)$ & 0.62 \\
\hline Bifidobacterium spp. & $10.4(0.5)$ & $10.3(0.3)$ & 0.50 \\
\hline C. leptum group & $11.6(0.7)$ & $11.4(0.3)$ & 0.41 \\
\hline C. coccoides group & $10.8(0.5)$ & $10.7(0.4)$ & 0.65 \\
\hline E. coli & $7.0(0.7)$ & $7.5(0.7)$ & 0.03 \\
\hline
\end{tabular}

Data $\left(\log _{10}\right.$ of $16 \mathrm{~S}$ rRNA, gene copy number) are presented as means (SD); $p$ value for paired $t$ test comparison between the interventions. ${ }^{\text {a }} \log _{10}$ of $16 \mathrm{~S}$ rRNA, gene copy number/g of wet fecal matter.

interventions for the Chaol index ( $p=0.87$ ) (Fig. 2), a measure of microbiome richness, or the Shannon diversity index $(p=0.46)$ (Fig. 2). Using either the Bray-Curtis dissimilarity (Fig. 2) index or UniFrac distance analysis, the fecal microbiome structure ( $\beta$ diversity) clustered primarily by enterotype and not by dietary intervention (Fig. 2 ; online suppl. material 3). There was no deviation of any participants from their enterotype, suggesting that the effect of meal timing was unable to supersede the effect of enterotypes. In differential analysis, the taxon relative abundance of 8 OTUs and 7 genera differed between the 2 interventions (Fig. 3). However, these significant effects vanished when analysis was corrected for multiple testing. Otherwise, the microbiome profile remained unaffected between the 2 dietary interventions at OTU (Fig. 4) and genus level assignments (online suppl. material 4).

\section{Absolute Quantification of Major Bacterial Groups}

The concentration (16S rRNA gene copy number/g of feces) of 5 major bacterial groups, representative of the 


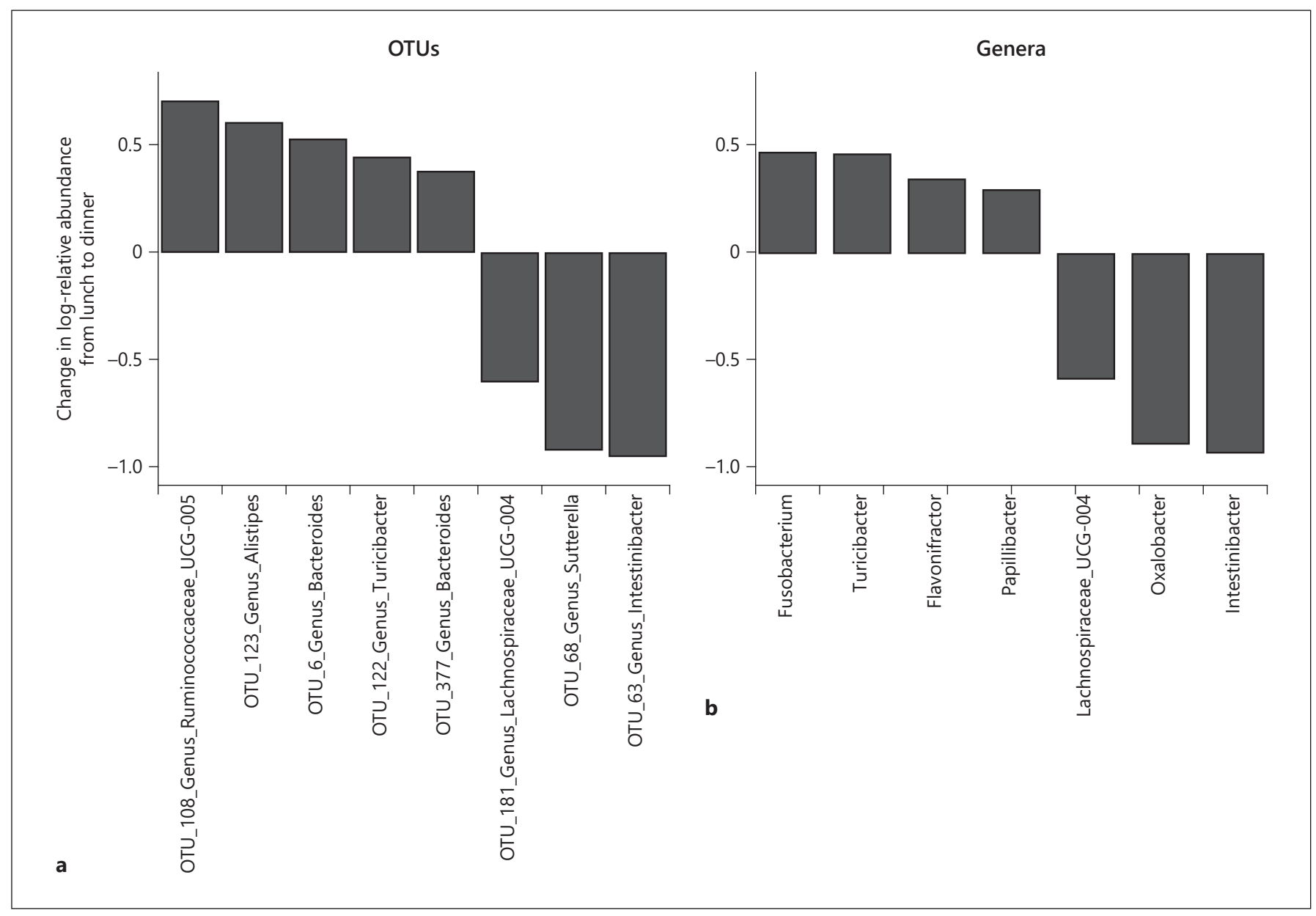

Fig. 3. Log-relative abundance significant differences between the 2 dietary interventions. a OTU level. b Genus level. OTU, operational taxonomic unit.

human gut microbiome, was quantified using qPCR. The time of main meal consumption did not influence significantly the fecal concentration of total bacteria or other bacterial groups except for $E$. coli, which was significantly higher after the large lunch intervention. This effect remained significant regardless of the approach used to express the data (Table 3).

\section{Fasting Blood Lipids}

The time at which participants consumed their main meal had no significant effect on fasting total cholesterol ( $p=0.97)$, LDL cholesterol $(p=0.87)$, HDL cholesterol $(p=0.75)$, or serum triglycerides $(p=0.86)$ (Fig. 5).

\section{Fasting Glucose, Insulin, and Insulin Resistance}

Fasted plasma glucose concentration did not differ between the 2 interventions $(p=0.77)$. Similarly, the time of main meal consumption had no significant effect on fasted plasma insulin concentrations $(p=0.77)$ or the homeostatic model assessment of insulin resistance (HOMA-IR) $(p=0.63)$ (Fig. 5).

\section{Low-Grade Systemic Inflammation}

Analysis of plasma IL-6 $(p=0.55)$ and TNF- $\alpha(p=$ 0.34 ) showed no difference in low-grade subclinical inflammation between the 2 dietary interventions. Similarly, no effect was observed for CRP which did not differ between the 2 interventions ( $p=0.78$ ) (Fig. 5). One participant had a raised CRP after the end of the second intervention due to a recent cold. Repetition of statistical analysis after exclusion of this participant did not change the difference in the mean effects observed between the 2 interventions. 

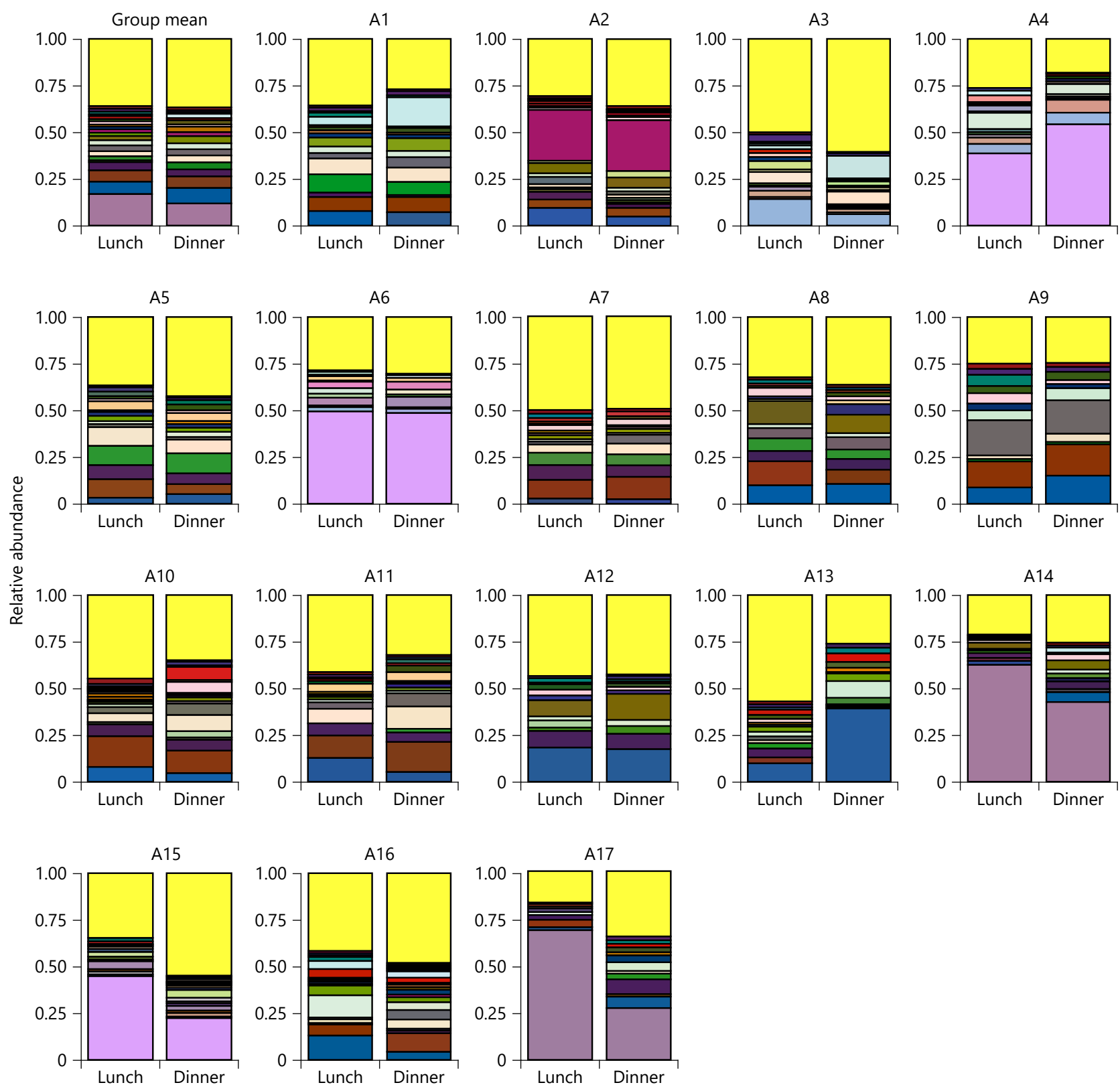

$\square$ OTU_1_Genus_Prevotella_9 $\square$ OTU_5_Genus_Bifidobacterium 口 OTU_7_Genus_Bacteroides OTU_16_Genus_Faecalibacterium $\square$ OTU_8_Genus_Agathobacter $\square$ OTU_6_Genus_Bacteroides $\square$ OTU 17 Genus Bacteroides $\square$ OTU_18_Genus_Collinsella $\square$ OTU_12_Genus_Bacteroides $\square$ OTU_10_Genus_Ruminococcus_
口OTU_2_Genus_Prevotella_7 - OTU_34_Genus_Blautia $\square$ OTU_39_Genus_Faecalibacterium $\square$ OTU_38_Family_Lachnospiraceae 2 OTU_27_Genus_Blautia
- OTU_26_Genus_Alistipes $\square$ Other

Fig. 4. Effect of the timing of main meal consumption on the relative abundance of the top 20 common OTUs group mean. Mean microbiome profile of all participants per dietary intervention. A1-A17, microbiome profile of each participant per dietary intervention; OTU, operational taxonomic units. 


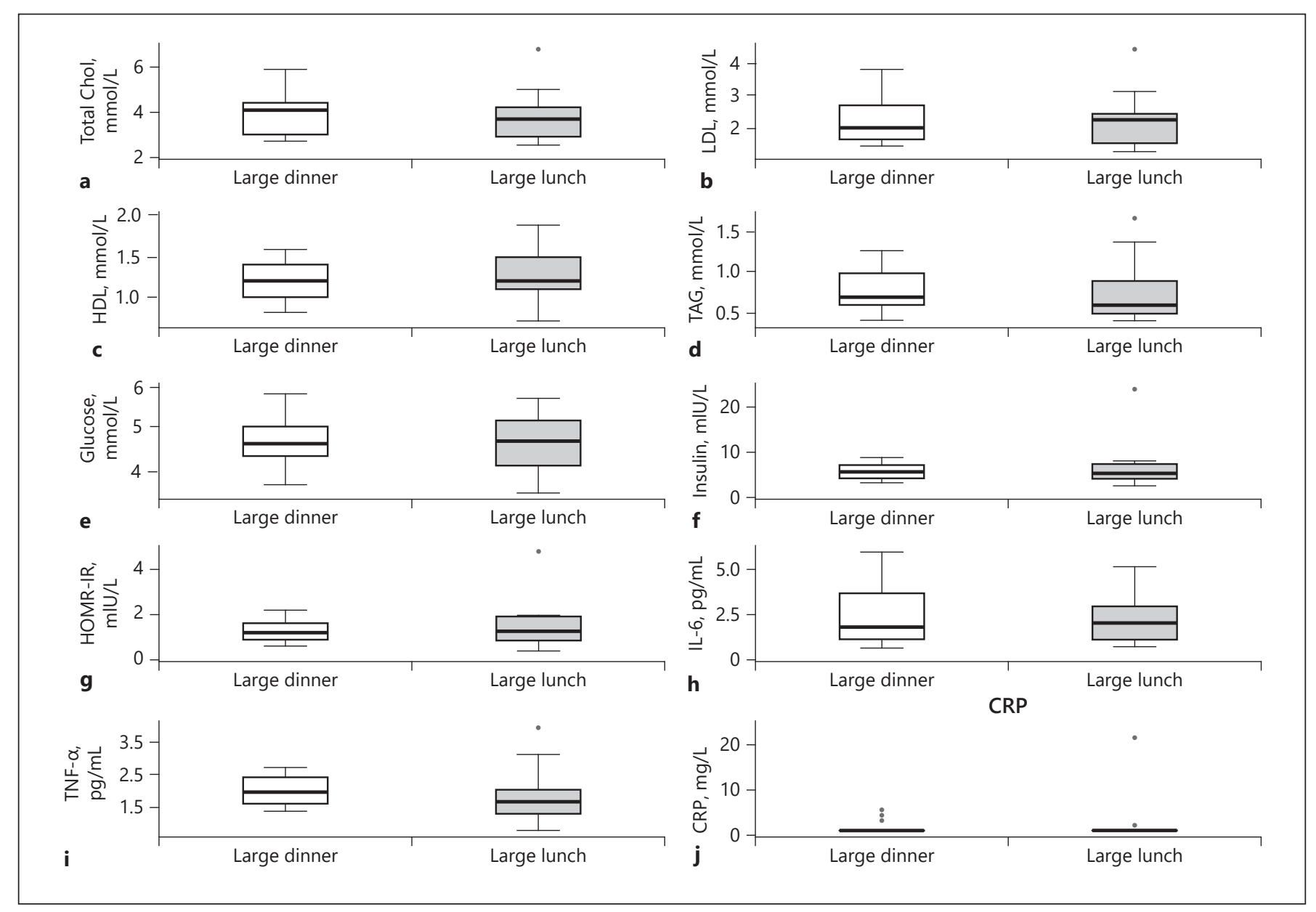

Fig. 5. Effect of timing of main meal consumption on blood biomarkers of cardiometabolic health. a Total cholesterol (mmol/L). b LDL cholesterol (mmol/L). c HDL cholesterol (mmol/L). d TAG (mmol/L). e Fasting glucose (mmol/L). f Insulin (mIU/L). g HOMA-IR (mIU/L). h IL-6 (pg/mL). i TNF-a (pg/mL). j CRP (mg/L). Box plots show means and quartiles. TAG, triglycerides; CRP, C-reactive protein. White boxplot indicates the large dinner intervention, and gray boxplot indicates the large lunch intervention.

\section{Discussion}

This study was set out to investigate the effect of the time of main meal consumption on the gastrointestinal microbiome and cardiometabolic factors of the host. Against our hypothesis, we found that the time at which the main meal was consumed had no acute effects on fecal $\mathrm{pH}$, form and texture of feces, fecal water content, total energy loss in feces, total fecal SCFA output, and the global microbial community structure or taxon relative abundance inferred using high-throughput sequencing. When we looked at the effect of the 2 interventions on the absolute concentration of dominant bacterial species, we observed a significant increase in the absolute concentration of $E$. coli after consumption of the main meal as lunch.
This effect is unlikely to be a random or spurious finding as it persisted when we expressed the amount of this species as the average concentration of all samples per participant, concentration, or total output over 3-day complete fecal sample collection. Interestingly, this signal was not replicated using high-throughput sequencing most likely due to different expression of data (absolute vs. relative abundance) and other counterbalancing changes. With the exception of the significant effect of our intervention on E. coli, these findings are against our hypothesis and those results presented previously in cross-sectional research [5]. It could be postulated that the E. coli increase was only apparent after consumption of the main meal at lunch time due to the long overnight fast between lunch and the next morning's breakfast, with only a small 
meal in between. Kaczmarek et al. [5] observed that overnight fasting was associated with relative abundance of several bacteria, including a nonsignificant trend $(p=$ $0.08)$ for Escherichia. Another study investigating the effects of the Western diet on the gastrointestinal microbiome of mice found that it caused an increase in fecal E. coli levels [15]. It is therefore possible that a similar response to the Western diet occurred in our participants.

Diet has been previously shown to influence fecal SCFA content [16], but evidence implicating main meal timing is scarce. We found no significant differences between the lunch and dinner meal interventions for any of the 10 SCFA tested. This observation agrees with previous cross-sectional research in which eating behavior (i.e., eating frequency, energy consumption early in the day, and overnight-fast duration) did not associate with the concentration of SCFA in spot stool samples despite a decline in the concentration of acetate, propionate, and butyrate of samples collected late during the day [5].

Similarly, we found no difference between the 2 interventions on blood biomarkers of CVD risk. This means that the time at which the main meal was consumed had no acute effects on blood CVD biomarkers such as blood lipids, insulin resistance, or low-grade inflammation, despite weight gain following the intervention with large dinner. Supporting the findings from this study, a recent weight loss study comparing the effects of a large lunch with a large dinner [9] found that although a decrease in blood lipids was noted for both groups and in parallel to their weight loss, the change in blood lipid level was not significantly different between the 2 interventions. Similarly, a crossover RCT investigating snacking times (10 a.m. vs. 11 p.m.) found that time of consumption of a high-fat, high-carbohydrate snack had no significant effects on serum triglycerides and HDL cholesterol, but late snacking produced a significant increase in total and LDL cholesterol [17].

Neither fasted blood insulin, glucose, nor HOMA-IR was responsive to different timing of the main meal. These findings are supported by previous research and a recent meta-analysis where snacking time had no effect on HOMA-IR, insulin, or glucose levels $[17,18]$. Contrary to this evidence, when meal times were delayed by $5 \mathrm{~h}$, plasma glucose rhythms were also delayed by approximately $5 \mathrm{~h}$ and average glucose concentration decreased [19]. Madjd et al. [9] also reported that a large lunch was associated with better insulin control when compared to individuals eating a large dinner. This result was, however, dependent on weight loss, which in the current study was not observed in the intervention with lunch as the main meal, and although weight gain was observed in

The Effect of Meal Timing on the Gut Microbiome the other group, the magnitude of this effect was very modest and perhaps too small to evoke an acute effect in young otherwise healthy subjects.

Two proinflammatory cytokines and CRP were measured here as markers of low-grade systemic inflammation, particularly as these markers are often elevated in obese individuals [20]. Although the origin of this lowgrade inflammation remains unknown, there is accumulating evidence to suggest that this might be due to the effect of diet on the gut microbiome and associated endotoxemia [2]. Our results suggest that time of main meal consumption is unlikely to be a contributor to low-grade inflammation, and this effect is unlikely to be associated with changes in the gut microbiome.

Bomb calorimetry was used on all stool samples from all participants to estimate intestinal absorption capacity [21] and the effect of the 2 interventions on the percentage of fecal water content and the Bristol Stool Scale as proxies of gastrointestinal motility. Nutrient load has previously been shown to be associated with changes to the microbiome and correlating with changes to energy loss in stool [22]. Nonetheless, this RCT is the first of its kind to investigate the associations between time of food consumption, energy content of stool, and microbiome changes and found no statistically significant association between time of main meal and cumulative energy content of fecal samples. Average fecal energy content met expected healthy values for both interventions [23].

This well-controlled study is not without its limitations. The study is of modest sample size, but the size effect and associated $p$ values observed suggest that this study is unlikely to suffer from loss of statistical power. Recruitment of more participants is unlikely to have altered the primary findings presented; therefore, extension of recruitment was deemed unnecessary. It is however possible that the discordant results for E. coli between $16 \mathrm{~S}$ rRNA sequencing and qPCR are due to lack of statistical power as well as the different expressions of data (relative abundance [\%] vs. absolute quantification [ $\log _{10} 16 \mathrm{~S}$ rRNA gene copies number/g of feces]).

We are also unable to comment on long-term effects that meal timing may have on the gut microbiome and cardiometabolic factors. With this in mind, the current study has several strengths. A robust study design was employed, with each participant acting as their own control. Furthermore, each participant had a personalized diet which equated their energy requirements and was based on their food preferences, and meals were provided to maximize compliance and accurate dietary intake assessment. 


\section{Conclusion}

This hypothesis testing study investigating the effects of the time of main meal consumption on the microbiome and the host found that main meal timing had minimal acute effects on cardiometabolic factors in the blood, intestinal absorption capacity, or on microbiome composition or activity. It is therefore unlikely that either a large lunch or a large dinner affects the diurnal rhythms of the gut microbiota and, by proxy, the onset of noncommunicable diseases influenced by the latter. In a presumptive causal pathway between timing of meal and risk of CVD onset, the gut microbiota is likely not to be implicated, at least in the short term. Future investigations should look to expand upon the findings of this study with longer duration of dietary interventions.

\section{Statement of Ethics}

The study received approval by the MLVS Research Ethics Committee at the University of Glasgow (Reference number: 200170046). All participants provided written informed consent and were reimbursed with GBP 100 shopping vouchers or a donation to a charity of their choice. The study was conducted accord- ing to the criteria set by the World Medical Association Declaration of Helsinki. Additionally, the study was registered with clinicaltrials.gov (NCT03949543).

\section{Conflict of Interest Statement}

None of the authors has any conflicts of interest to disclose relevant to this study.

\section{Funding Sources}

Miss Katrina Ballantyne received a summer undergraduate student bursary by the Rank Prize Funds. Dr. Ben Nichols is partially funded by the Biotechnology and Biological Sciences Research Council (BB/R006539/1).

\section{Author Contributions}

R.T., C.S.L., and K.B. conducted the clinical aspects of the research and data collection; R.T.P., D.M., A.V., M.R., and P.G. conducted the laboratory analysis of samples; B.N. performed the statistical analysis of the data and microbiome bioinformatics; K.G. designed and supervised the research, wrote the paper with the support of R.T.P. and D.M., and has the primary responsibility for the final content.

\section{References}

1 Quince C, Ijaz UZ, Loman N, Eren AM, Saulnier D, Russell J, et al. Extensive modulation of the fecal metagenome in children with Crohn's disease during exclusive enteral nutrition. Am J Gastroenterol. 2015 Dec; 110(12):1718-30.

2 Khan MJ, Gerasimidis K, Edwards CA, Shaikh MG. Role of gut microbiota in the aetiology of obesity: proposed mechanisms and review of the literature. J Obes. 2016; 2016:7353642.

3 Singh RK, Chang HW, Yan D, Lee KM, Ucmak D, Wong K, et al. Influence of diet on the gut microbiome and implications for human health. J Transl Med. 2017 Apr 8;15(1):73.

4 Larsen N, Vogensen FK, van den Berg FW, Nielsen DS, Andreasen AS, Pedersen BK, et al. Gut microbiota in human adults with type 2 diabetes differs from non-diabetic adults. PLoS One. 2010 Feb 5;5(2):e9085.

5 Kaczmarek JL, Musaad SM, Holscher HD. Time of day and eating behaviors are associated with the composition and function of the human gastrointestinal microbiota. Am J Clin Nutr. 2017 Nov;106(5):1220-31.

6 Leone V, Gibbons SM, Martinez K, Hutchison AL, Huang EY, Cham CM, et al. Effects of diurnal variation of gut microbes and high-fat feeding on host circadian clock function and metabolism. Cell Host Microbe. 2015;17(5): 681-9.

7 Voigt RM, Forsyth CB, Green SJ, Engen PA, Keshavarzian A. Circadian rhythm and the gut microbiome. Int Rev Neurobiol. 2016; 131:193-205.

8 Thaiss CA, Zeevi D, Levy M, Zilberman-Schapira G, Suez J, Tengeler AC, et al. Transkingdom control of microbiota diurnal oscillations promotes metabolic homeostasis. Cell. 2014 Oct 23;159(3):514-29.

9 Madjd A, Taylor MA, Delavari A, Malekzadeh R, Macdonald IA, Farshchi HR. Beneficial effect of high energy intake at lunch rather than dinner on weight loss in healthy obese women in a weight-loss program: a randomized clinical trial. Am J Clin Nutr. 2016 Oct; 104(4):982-9.

10 Almoosawi S, Vingeliene S, Karagounis LG, Pot GK. Chrono-nutrition: a review of current evidence from observational studies on global trends in time-of-day of energy intake and its association with obesity. Proc Nutr Soc. 2016 Nov;75(4):487-500.

11 Collado MC, Engen PA, Bandín C, CabreraRubio R, Voigt RM, Green SJ, et al. Timing of food intake impacts daily rhythms of human salivary microbiota: a randomized, crossover study. FASEB J. 2018 Apr;32(4):2060-72.
12 David LA, Maurice CF, Carmody RN, Gootenberg DB, Button JE, Wolfe BE, et al. Diet rapidly and reproducibly alters the human gut microbiome. Nature. 2014;505(7484):55963.

13 Svolos V, Hansen R, Nichols B, Quince C, Ijaz UZ, Papadopoulou RT, et al. Treatment of active Crohn's disease with an ordinary foodbased diet that replicates exclusive enteral nutrition. Gastroenterology. 2019 Apr;156(5): 1354-67.e6.

14 Gerasimidis K, Bertz M, Hanske L, Junick J, Biskou O, Aguilera M, et al. Decline in presumptively protective gut bacterial species and metabolites are paradoxically associated with disease improvement in pediatric Crohn's disease during enteral nutrition. Inflamm Bowel Dis. 2014 May;20(5):86171.

15 Martinez-Medina M, Denizot J, Dreux N, Robin F, Billard E, Bonnet R, et al. Western diet induces dysbiosis with increased $\mathrm{E}$ coli in CEABAC10 mice, alters host barrier function favouring AIEC colonisation. Gut. 2014 Jan; 63(1):116-24.

16 Scott KP, Gratz SW, Sheridan PO, Flint HJ Duncan SH. The influence of diet on the gut microbiota. Pharmacol Res. 2013 Mar;69(1): $52-60$. 
17 Hibi M, Masumoto A, Naito Y, Kiuchi K, Yoshimoto Y, Matsumoto M, et al. Nighttime snacking reduces whole body fat oxidation and increases LDL cholesterol in healthy young women. Am J Physiol Regul Integr Comp Physiol. 2013 Jan 15;304(2): R94-101.

18 St-Onge MP. Sleep-obesity relation: underlying mechanisms and consequences for treatment. Obes Rev. 2017 Feb;18(Suppl 1):34-9.

19 Wehrens SMT, Christou S, Isherwood C, Middleton B, Gibbs MA, Archer SN, et al.
Meal timing regulates the human circadian system. Curr Biol. 2017 Jun 19;27(12):1768e3.

20 Ramos EJ, Xu Y, Romanova I, Middleton F, Chen C, Quinn R, et al. Is obesity an inflammatory disease? Surgery. 2003;134(2):32935.

21 Wierdsma NJ, Peters JH, van Bokhorst-de van der Schueren MA, Mulder CJ, Metgod I, van Bodegraven $\mathrm{AA}$. Bomb calorimetry, the gold standard for assessment of intestinal absorption capacity: normative values in healthy ambulant adults. J Hum Nutr Diet. 2014 Apr; 27(Suppl 2):57-64.

22 Jumpertz R, Le DS, Turnbaugh PJ, Trinidad C, Bogardus C, Gordon JI, et al. Energy-balance studies reveal associations between gut microbes, caloric load, and nutrient absorption in humans. Am J Clin Nutr. 2011 Jul; 94(1):58-65.

23 Atwater WO, Rosa EB. A new respiration calorimeter and experiments on the conservation of energy in the human body. I. Physical Review (Series I). 1899 Nov 1;9(3):129-63. 